\title{
Lumen
}

Selected Proceedings from the Canadian Society for Eighteenth-Century Studies

\section{La petite maison : un concept architectural au service d'une sociabilité nouvelle}

\section{Claire Ollagnier}

Volume 35, 2016

URI : https://id.erudit.org/iderudit/1035919ar

DOI : https://doi.org/10.7202/1035919ar

Aller au sommaire du numéro

Éditeur(s)

Canadian Society for Eighteenth-Century Studies / Société canadienne d'étude du dix-huitième siècle

ISSN

1209-3696 (imprimé)

1927-8284 (numérique)

Découvrir la revue

Citer cet article

Ollagnier, C. (2016). La petite maison : un concept architectural au service d'une sociabilité nouvelle. Lumen, 35, 37-46. https://doi.org/10.7202/1035919ar d'utilisation que vous pouvez consulter en ligne. 


\title{
La petite maison : un concept architectural au service d'une sociabilité nouvelle
}

\author{
Claire Ollagnier \\ Université Paris I Panthéon-Sorbonne
}

Dans la première moitié du XVIII ${ }^{\mathrm{e}}$ siècle ${ }^{1}$, l'idée d'un lieu situé à l'abri de tous les regards où le libertin pourrait s'adonner à ses activités licencieuses s'est répandue à travers la littérature romanesque et théâtrale. Peu à peu, les rapports de police et les chroniques scandaleuses regorgent d'anecdotes piquantes: ainsi naît le mythe de la petite maison. Celle-ci est définie dans le Cours d'architecture de JacquesFrançois Blondel ${ }^{2}$. Le théoricien l'intègre dans une typologie plus générale de l'habitat domestique et lui associe alors une suite de caractères propres et différenciables, en d'autres termes un programme. Tout d'abord la petite maison se situe en périphérie urbaine. Dans un monde où la vie de l'homme de qualité s'organise entre son hôtel urbain et son château en campagne, l'espace situé en marge du centre de la capitale permet à l'imaginaire de s'exprimer comme nulle part ailleurs et offre au propriétaire un moyen de déroger quelque peu aux exigences de la bienséance ${ }^{3}$. Par ailleurs, aucun statut particulier ne

1. L'expression «petite maison» semble être en usage depuis la seconde moitié du XVII ${ }^{\mathrm{e}}$ siècle; Madeleine de Scudéry qualifie ironiquement le château de Versailles de «la petite maison du plus grand des rois» (La promenade de Versailles, dédiée au roi, Paris, 1669, cité dans Katarina Krause, Die Maison de plaisance, Landhäuser in der Île-de-France (166o-1730), Berlin, Deutscher Kunstverlag, 1996, p. 33).

2. Jacques-François Blondel, Cours d'architecture contenant les leçons données en 1750 et les années suivantes, Paris, Desaint, 1771, vol. II, p. 251.

3. Voir Antoine Lilti, Le monde des salons. Sociabilité et mondanité à Paris au XVIII siècle, Paris, Fayard, 2005, p. 221. Si la société est encore hantée par l'idéal aristocratique et structurée par le rôle central de la cour, elle est également travaillée par les remises en cause du privilège nobiliaire. 
semble nécessaire à son acquisition : seuls les moyens financiers, le bon goût du propriétaire et son intérêt pour la retraite (quelles qu'en soient les motivations) peuvent conduire à investir dans cette zone encore peu bâtie. Ainsi la commande émane-t-elle de personnalités issues d'une élite parisienne, cultivée et cosmopolite, nourrissant de prime abord un intérêt particulier pour les jardins, l'architecture et les arts en général.

Tous les quartiers périphériques de la capitale se couvrent alors peu à peu d'édifices aux allures diverses mais répondant à ces mêmes critères. Des sommes extravagantes sont employées à bâtir les demeures les plus novatrices. Les artistes trouvent dans ce programme la liberté nécessaire au véritable épanouissement de leur art, expérimentant des techniques et des procédés architecturaux inédits ${ }^{4}$. Du point de vue des usages, ces demeures se distinguent par des pratiques particulières: ainsi la petite maison est-elle souvent associée à un certain nombre d'activités libertines; mais qu'en est-il vraiment?

Les témoignages contemporains dressent un portrait plus contrasté des pratiques sociales qui se jouent en périphérie urbaine : aristocrates, financiers, grands bourgeois, et leur suite de femmes entretenues et d'artistes à la mode y partagent leur quête de divertissements physiques mais également intellectuels. Ce sont ces pratiques sociales que nous allons exposer ici. Pratiques qui, si elles ne concernent qu'une élite parisienne de haute volée, n’en demeurent pas moins révélatrices d’une pensée progressiste qui tend à s'exprimer. Ainsi peut-on percevoir, derrière l'apparente quête de légèreté que suscite l'attrait du divertissement, l'émergence d'une conscience accrue de l'individu et la maturation d'une révolution sociale, dont l'architecture est un témoin non négligeable.

4. Nous renvoyons le lecteur à notre récente étude de synthèse : Claire Ollagnier, Petites maisons suburbaines au XVIII siècle, du pavillon d'agrément au pavillon d'habitation, Bruxelles, Mardaga, 2016. Par ailleurs, un certain nombre de publications se rapporte à la petite maison sous d'autres dénominations; sur les folies voir: Gilles-Antoine Langlois, Les folies françaises, 1700-180o, Lille, Université de Lille, Mémoire de thèse, 1990; "Folies au XVIII siècle", Sites et monuments, 117, 1987, p. 6-11; De Bagatelle à Monceau, 1778-1978, les folies du XVIII siècle à Paris, Paris, Musée Carnavalet, 1978; Marcel Fouquier, Paris au XVIII siècle: ses folies, Paris, 1912; Gaston Capon, Les petites maisons galantes de Paris au XVIII ${ }^{e}$ siècle. Folies, maisons de plaisance et vide-bouteilles, d'après les documents inédits et des rapports de police, Paris, H. Daragon, 1902. 


\section{Les divertissements intérieurs}

Comme l'expose Blondel dans sa définition, la petite maison constitue avant tout une retraite occasionnelle consacrée au délassement, «au plaisir et à la liberté $»$. Pour la bonne société capable de s'offrir de telles demeures, il s'agit avant tout d'échapper à l'ennui que provoque l'oisiveté. Or, ainsi que l'a montré Antoine Lilti: «le monde où l'on s'ennuie et le monde où l'on s'amuse sont les deux faces d'un même phénomène ${ }^{6} »$.

Dans les témoignages contemporains, les références aux parties de débauche se déroulant dans la petite maison ne manquent pas: petits soupers enivrés où les femmes s'essaient et s'échangent sans aucune gêne, représentations théâtrales privées mettant en scène les plus grandes comédiennes parisiennes dans leur plus simple appareil. La licence prétendue des échanges qui se nouent dans la petite maison fait les «choux gras» des mémorialistes et des journalistes. La littérature érotique aime également à placer ses personnages autour d'une belle table qui invite autant aux plaisirs charnels qu'un boudoir ou un cabinet dérobé. Ainsi peut-on lire dans Angola, histoire indienne sans vraisemblance, de La Morlière:

Lune [des pièces de la petite maison] destinée aux plaisirs de la table, paraissait garnie avec une profusion délicate, de tout ce que le goût le plus raffiné a pu imaginer en faveur de cette sensualité?.

Est-ce ce genre de repas fins que goûtait le duc de Laval dans la petite salle à manger située au rez-de-chaussée de sa maison du boulevard Montparnasse et précisément nommée «cabinet secret pour donner à manger ${ }^{8} »$ ? C'est probable.

Sous la plume d'Élisabeth Vigée-Lebrun pourtant, la petite maison se présente davantage comme le lieu de rencontre de l'intelligentsia parisienne. Ainsi relate-t-elle les «jeudis», véritables «salons», de M. Boutin :

5. Jacques-François Blondel, Cours d'architecture, op. cit., vol. II, p. 251.

6. Antoine Lilti, Le monde des salons, op. cit., p. 225.

7. Jacques-Rochette de La Morlière, Angola, histoire indienne sans vraisemblance [1747], dans Romans libertins du XVIII siècle, éd. de R. Trousson, Paris, Robert Laffont, coll. «Bouquins », 1999, p. 415 .

8. Voir Jean-Charles Krafft et Nicolas Ransonnette, Plans, coupes, élévations des plus belles maisons ¿ des hôtels construits à Paris \& dans les environs entre 1771 et 1802, Paris, s.n., 1802, pl. 41 [légendes]. 
Nous étions au plus douze personne à table [...]. [Ces dîners] avaient lieu dans cette charmante maison de M. Boutin, placée sur la hauteur du magnifique jardin qu'il avait nommé Tivoli : à cette époque la rue de Clichy n'était pas encore bâtie, et quand on se trouvait là, au milieu d'arbres superbes qui formaient de grandes et belles allées, on pouvait se croire tout à fait à la campagne; je puis même dire que cette belle habitation me semblait un peu trop isolée $[\ldots]^{9}$.

La table constitue donc essentiellement un lieu particulièrement propice à l'échange entre amis. Chez $\mathrm{M}^{\text {lle }}$ Guimard à la Chaussée d'Antin, un dispositif spectaculaire est mis en place : la salle à manger est associée à un jardin d'hiver. L'ensemble est ainsi décrit par Horace Walpole :

Le seul exemple de luxe dont on parle est dû à $\mathrm{M}^{\text {lle }}$ Guimard [...] qui est en train de se bâtir un palais; autour de la salle à manger sont deux fenêtres ouvrant sur une serre chaude qui produira des fleurs tout l'hiver ${ }^{10}$.

Par ailleurs, dans certaines demeures, la salle à manger offre également la possibilité de se distraire et possède des tables à jouer ${ }^{11}$. Alors que certains goûtent aux plaisirs culinaires, d'autres viennent seulement jouer au Tric Trac ou bavarder au coin du feu.

Les petites maisons disposaient le plus souvent d'un appartement des bains s'ouvrant sur le jardin pour permettre à l'individu de jouir, au bain, d'un paysage varié et d'agrémenter son séjour voluptueux d'une rêverie bucolique. Peut-on alors parler de lieu de sociabilité? Les appartements des bains conçus pour les femmes de scène laisse présager des usages particuliers. Chez $\mathrm{M}^{\text {lle }}$ Dervieux, un appartement très vaste assorti d'un boudoir semble parfaitement adapté au bain collectif, alors que dans la demeure de $\mathrm{M}^{\text {lle }}$ Guimard la situation de l'appartement des bains, à l'entrée de la maison, annonce avec éloquence les usages qui lui sont associés. Directement accessible depuis le vestibule, il saffiche comme un espace de réception - voire de passage - en tant que tel. On peut donc aisément supposer que certains appartements

9. Elisabeth Louise Vigée-Lebrun, Souvenirs, Paris, Éditions des femmes, 2005, t. II, p. 243.

10. Extrait d'Horace Walpole, cité par Daniel Rabreau, Claude-Nicolas Ledoux (1736-18o6), L'architecture ou les fastes du temps, Paris/Bordeaux, William Blake \& Co/Art\&Arts, Annales du Centre Ledoux t. III, 2000, p. 79-80.

11. Donald Spinelli, Inventaire après décès de Beaumarchais, Paris, Champion, 1997, p. 39-49. 
des bains aient été davantage conçus pour l'agrément d'une société nombreuse que pour le délassement du seul propriétaire.

Au-delà des sociabilités liées au repas et des pratiques a priori plus intimes qui se jouent dans les appartements des bains ou les boudoirs, les petites maisons témoignent de la place toujours plus grande accordée au théâtre. Il est vrai que, durant les trente dernières années du siècle, les transgressions se multiplient sur les scènes privées et notamment chez certaines comédiennes. Ces représentations étaient animées par des amateurs et des professionnels et il est avéré que, dans un contexte privé, la distinction entre les spectateurs et les acteurs s'estompe au profit du lien de sociabilité ${ }^{12}$. Néanmoins, pour les chercheurs que nous sommes, les informations sur les représentations de théâtre de société sont assez difficiles à obtenir ${ }^{13}$. Les rares journaux d'époque qui ont tenté de relater ces évènements ont rapidement été confrontés à la censure ${ }^{14}$ : on n'en parle pas sans y avoir été autorisé! On sait toutefois que les pièces représentées se répartissent en trois catégories: le répertoire classique, les productions récentes et les pièces interdites sur les scènes officielles. Ces dernières se distinguent par leur caractère érotique - voire franchement graveleux - mais aussi parce qu'elles dérogent à la bienséance de l'ordre établi.

Chez $\mathrm{M}^{\text {lle }}$ Guimard, on pouvait fréquemment assister à des productions récentes, productions qui se poursuivaient bien souvent par des orgies très bien orchestrées par la maîtresse de maison dans son vaste théâtre conçu par Ledoux. Le Mariage de Figaro de Beaumarchais figure également dans la catégorie des pièces interdites mais pour d'autres raisons. Écrite dans les années 1778-1780, la pièce est plusieurs fois censurée et refusée à la représentation. Les Mémoires secrets - dont la publication rétroactive permet une liberté de ton qui serait mal venue de la part d'un quotidien - annoncent toutefois qu'une première représentation a eu lieu le 27 septembre 1783 sur une scène privée. La pièce se joue en effet à Gennevilliers, dans la propriété du comte de

12. Antoine Lilti, Le monde des salons, op. cit., p. 254.

13. Le théâtre de société est un sujet qui a fait l'objet de plusieurs études récentes; un colloque intitulé Les théatres de société au XVIII siècle s'est notamment déroulé en 2005 à Versailles. Ces études se concentrent le plus souvent sur l'analyse des répertoires choisis par les amateurs pour être joués sur les scènes non-officielles et sur celle de la composition des troupes particulières.

14. Voir à ce sujet Le Journal de Paris, n 107 (17 avril 1777), nº 109 (19 avril 1777), et $n^{\circ} 110$ (20 avril 1777). 
Vaudreuil - ami du comte d'Artois. Cette représentation fit couler beaucoup d'encre : plusieurs mémorialistes en font mention, $\mathrm{M}^{\mathrm{me}}$ VigéeLebrun, et M. Fleury notamment. Grâce à la correspondance de Beaumarchais, on sait qu'à la suite de cette représentation privée, une représentation officielle est enfin autorisée. Celle-ci a lieu le 27 avril 1784 au Théâtre Français et est longuement mentionnée dans la presse de l'époque. Le Journal de Paris publie de nombreux détails sur les rôles des différents acteurs, détails grâce auxquels on peut constater qu'il s'agit de la même troupe que lors de la représentation de Gennevilliers. On est donc face à une représentation particulière qui n'obéit pas vraiment aux règles de théâtre de société : il ne s'agit pas du bon divertissement d'une troupe de particuliers, mais bien d'une action menée dans l'ombre. Il est évident que la première de Gennevilliers représentait une avancée extraordinaire vers la représentation officielle.

\section{Les extérieurs: du spectaculaire au poétique}

Outre les divers espaces intérieurs que nous venons de présenter et qui permettent à un groupe social éclairé d'interagir et d'échanger, la petite maison se distingue par l'importance donnée à ses jardins. Jusqu'au milieu du XVIII ${ }^{\mathrm{e}}$ siècle, le jardin se déduisait de l'architecture. Mais les nombreux débats qui émergent alors conduisent les concepteurs à l'envisager sous un angle nouveau: la demeure ne se conçoit bientôt plus sans égard pour son environnement naturel, et le jardin se soustrait à la vraie nature. Cette évolution s'accompagne d'une transformation des pratiques et des usages.

Les divertissements qui ont cours à l'intérieur de la demeure se poursuivent bien souvent dans les jardins dont la composition est de plus en plus savante. Les créateurs de jardin se plaisent en effet peu à peu à introduire des surprises et des extravagances par le biais de «fabriques» à l'apparence et aux destinations diverses ${ }^{15}$. Ainsi convientil de citer le dispositif mis en scène dans le jardin du duc de Chartres à Monceau: au fond d'une grotte profonde, le propriétaire, par le moyen d'un cordon, peut prévenir les musiciens cachés dans une pièce au-dessus pour que ceux-ci commencent à jouer; la musique pénètre

15. Sur les fabriques, voir Monique Mosser, «Les architectures paradoxales ou petit traité des fabriques", Histoire des jardins de la Renaissance à nos jours, dans Monique Mosser et Georges Teyssot (dir.), Paris, Flammarion, 2002 [1991], p. 259-276. 
alors par les lézardes de la roche et vient surprendre agréablement les convives qui pénètrent dans la grotte. Dans les jardins de Baudard de Saint-James à Neuilly, la fabrique la plus remarquée consiste en une sorte de grotte artificielle. Il ne s'agit pas là d'un simple décor pittoresque; le rocher dissimule en réalité un dispositif sophistiqué: une salle de bain souterraine, judicieusement conçue sous le minéral massif. Chez Beaumarchais, au faubourg Saint-Antoine, les plaisirs de la table se poursuivent au jardin. Le célèbre écrivain y fait en effet construire un temple de Bacchus destiné aux collations.

Tous ces dispositifs sont conçus dans le but de provoquer la surprise auprès des visiteurs, quelques amis ou connaissances triées «sur le volet». Surprise bien souvent amplifiée par quelques divertissements éphémères et attractifs: feux d'artifice et jeux d'eau. C'est aussi dans les jardins que l'on installe des jeux de bague et des balançoires ${ }^{16}$. Lengouement est tel que beaucoup de ces jardins sont ouverts au public sur présentation d'un billet, parfois payant: Bagatelle est accessible plusieurs fois par semaine, de même que la Chartreuse de Beaujon, ou au Tivoli de Boutin; Beaumarchais distribue des billets à tous ceux qui lui en font la demande, y ajoutant parfois un mot de sa main ${ }^{17}$.

Ces divertissements collectifs sont également complétés par de nouveaux passe-temps qui se rapportent aux sciences et à l'histoire naturelle, composantes essentielles de tout loisir honorable. À cette époque, nombre de témoignages révèlent la passion du public pour l'histoire naturelle, intérêt complété par le jardinage et l'agriculture, que même les plus lettrés pratiquent aisément. Par l'intermédiaire du Journal de Paris, les amateurs font part de leurs observations, de leurs interrogations; ils cherchent des réponses et espèrent obtenir de l'aide: comment soigner un arbre malade? où planter telle ou telle variété de plante pour qu'elle se développe le mieux? Que faire du surplus de récolte de fruit?... Tous écrivent de leur «campagne», de leur «maison près de Paris ». Au-delà du simple émerveillement provoqué par les beautés de la nature, on assiste donc à un véritable engouement scientifique, à une curiosité et à une volonté de comprendre les phénomènes naturels et biologiques qui font partie de l'environnement

16. Gilles-Antoine Langlois, Folies, tivolis et attractions. Les premiers parcs de loisirs parisiens, Paris, Délégation à l'action artistique, 1991, p. 31.

17. Maurice Lever, Pierre-Augustin Caron de Beaumarchais, t. III «Dans la tourmente (1785-1799) », Paris, Fayard, 2003, p. 155. 
des propriétaires. On peut alors s'interroger : n'y a-t-il pas une incompatibilité entre la mondanité aristocratique, superficielle et brillante, et la recherche scientifique, sévère et profonde? Bien au contraire, le roi lui-même montre l'exemple au Petit Trianon. De plus, on sait avec certitude que certains propriétaires, aristocrates ou bourgeois, se livrent à des expériences dans leurs jardins: culture de plantes nouvelles, création de prairies artificielles, etc. ${ }^{18}$ Ainsi l'agriculture devient-elle un passe-temps pour l'élite éclairée soucieuse de montrer qu'elle participe au progrès de son siècle. Les cabinets d'histoire naturelle prennent alors le relais des cabinets de curiosité déjà fort à la mode et il convient à présent de les accompagner d'un dispositif spectaculaire: Baudard de Saint-James élève des serres disposées en croix autour de son cabinet d'histoire naturelle, alors que celui du comte d'Artois à Bagatelle, précisément décrit par Thiéry, est nommé «Pavillon du Philosophe ${ }^{19}$ ».

Alors que l'influence rousseauiste est à son comble, la volonté des élites d'opérer une symbiose entre l'art des jardins, lié à l'embellissement des demeures de l'élite, la botanique - longtemps tenue pour la partie la plus noble de l'agriculture - et l'agronomie, s'impose comme une nécessité. Il s'agit de faire en sorte que «le jardin concilie avantages économiques, plaisir esthétique et vertu morale ${ }^{20} »$. Si les esprits s'orientent vers la réunion de l'utile et de l'agréable, la petite maison apparaît à nouveau comme une réponse, un lieu de rencontre entre la théorie et la pratique. On s'est beaucoup moqué des dames de cour déguisées en fermières à une époque où les laiteries et autres fermes ornées s'installent dans les jardins des petites maisons. Mais, étant donné toutes les vertus attribuées au monde rural, il n'est pas étonnant qu'on cherche à le créer partout où il est absent, à en imiter le naturel et la simplicité21. Ainsi on voit l'aménagement de laiteries réservées à la dégustation des produits frais ${ }^{22}$ : celle de Simon Gabriel Boutin, rue Saint-Lazare, réalisée avant 1775, celle du duc de Chartres, au parc

18. Voir Catherine Clavilier, Cérès et le laboureur, Paris, Éditions du patrimoine, coll. «Temps et espace des arts », 2009, p. 66-67.

19. Voir Luc-Vincent Thiery, Guide des amateurs et des étrangers voyageurs à Paris, Paris, Chez Gattey, 1787, t. I, p. 25-30.

20. Jacques Delille, Les jardins, ou l'art d'embellir les paysages, Paris, F.-A. Didot, 1782, "Avertissements ».

21. Catherine Clavilier, Cérès et le laboureur, op. cit., p. 91.

22. Voir Mona Oulami, La laiterie au jardin, 1723-1814, Paris, Université Paris I, Mémoire de Master II recherche, 2010. 
Monceau, vraisemblablement construite entre 1772 et 1778 (la maison rustique du Meunier est en fait une laiterie à l'intérieur) ou encore la laiterie que Bélanger construisit dans un labyrinthe souterrain de la Folie Saint-James en $1778 \ldots$

En réalité, ces édifices sont autant conçus pour leur vertu divertissante que pour leur utilité, et offrent un excellent exemple d'artificialité et d'utilitarisme ${ }^{23}$. Dans cette perspective, ces comportements apparaissent moins comme la manifestation un peu sotte d'une futilité oisive, voire d'une indécence, que comme l'expression d'une société où l'imaginaire se confond aisément avec la réalité. Le fait d'intégrer le jardin d'agrément à une réflexion économique plus large apparaît donc comme un moyen de le moraliser. La seule jouissance du propriétaire ne suffit plus à fonder une théorie du jardin et à légitimer son existence; il faut qu'il obéisse à des fins productives, qu'il contribue à l'enrichissement collectif - conformément à l'idéal de physiocratie qui se développe alors.

Alors que l'on pressent une volonté de s'amuser tout en s'adonnant à des activités sérieuses et utiles, il est possible de s'interroger: est-ce un moyen de légitimer le divertissement ou au contraire de voiler les véritables préoccupations d'un groupe social soucieux de son apparence? En d'autres termes, cette élite en prise avec les contradictions d'une monarchie finissante cache-t-elle ses inquiétudes derrière une apparente légèreté? De fait, la plupart des jardins à fabriques ont une portée symbolique et même initiatique forte. À l'heure où la francmaçonnerie se développe et offre une possible voie de régénération sociale, de trop rares témoignages viennent confirmer ce que l'observation d'un bon nombre de jardins de petites maisons nous laisse supposer $^{24} \ldots$ en effet, nous n'avons à ce jour aucune preuve que des rituels maçonniques se soient déroulés dans une petite maison (même celle du duc de Chartres, grand-maître du Grand Orient de France depuis 1773). Néanmoins, les jardins symboliques apparaissent souvent comme la manifestation à peine voilée d'une pensée nouvelle, progressiste, également revendiquée par les loges maçonniques: jardin du duc de Chartres à Monceau, rocher de la Folie Saint-James...

23. Catherine Clavilier, Cérès et le laboureur, op. cit., p. 91.

24. Voir «Promenade étrange de Sir William Beckford en compagnie de Ledoux», cité dans Michel Gallet, «Ledoux et Paris », Cahiers de La Rotonde, Paris, Rotonde de la Villette, 1979, vol. III, p. 146-152. 
Certes, les références aux parties de débauche se déroulant dans la petite maison ne manquent pas. Le plaisir et la liberté, c'est bien de cela dont il s'agit ici : la jouissance sous toutes ses formes doublée d'une liberté d'action quasiment impossible ailleurs. À travers le prisme des loisirs, la petite maison apparaît comme un abri où de nouvelles pratiques de sociabilité vont pouvoir se développer. Les petits soupers intimes et les représentations théâtrales de plus ou moins grande ampleur témoignent bien, au sein d'une élite éclairée, de mutations qui seront très probablement à l'origine d'une révolution sociale. Car ici la légèreté n'est qu'apparente et la portée de ces nouvelles pratiques qui tendent à se poursuivre dans les jardins, portée idéologique et politique, ne doit pas être sous évaluée.

Les usages qui se développent au sein de la petite maison se présentent in fine comme une volonté de joindre l'utile à l'agréable, et les notions subversives qui en découlent s'inscrivent bien évidemment dans le même élan transgressif. Ainsi la quête de régénération passe bien souvent par la mise en scène de divertissements futiles, aboutissant ainsi à une vision factice ou artificielle de ce que fut, à l'aube de la Révolution, la vie et l'engagement politique des élites. 